\title{
Serviceable Website for Enjoying Food through Online
}

\author{
Avinash C. \\ Department of Computer \\ Science \\ VIT University \\ Vellore -15
}

\author{
Hasritha $P$. \\ Department of Computer \\ Science \\ VIT University \\ Vellore -15
}

\author{
Abhinay J. \\ Department of Computer \\ Science \\ VIT University \\ Vellore -15
}

\begin{abstract}
Generally, all the restaurants want to be famous for increasing the number of users. Online acts as a vital role to improve the popularity of hotels. According to that this project is developed for reputed restaurants to providing online for reaching huge number of users. Online business is growing fast so lots of profession is providing online services for customers. Particularly food service websites are very helpful for ordering food to users. It will take a minimum amount of time. In this project we launch efficacious website for hotels to developing user's interaction. By this, the user can order their food without any inconvenient. This will increase the number of customers to the hotels.
\end{abstract}

\section{Keywords}

Enjoin, Tariff, Mesh, Feedback, Tidy

\section{INTRODUCTION}

$\mathrm{N}$ presents of fast food, many restaurants have selected to concentrate on rapid composition and immediate distribution of requests rather than subscribing an affluent consumes experience. As late as in last few days, all of these conveyance requests were located in excess of the telephone, other than there are a lot of demerits to this scheme, inclusive of the nuisance of the creature require to contain a objective carbon copy of the set of choices, absence of a visual justification that the request was placed perfectly, and the essential for an informal eatery to have a member of the workforce responding the extensions and taking enjoins. The challenges encountered by the existing system serve as major negative aspects to the acknowledgment of effectiveness and customer approval. The experience of ordering in most fast food restaurants is not enjoyable for the consumers. Trade will have to make long queues before insertion their guidelines especially during reach your zenith hours and then the ordering staff will trace customer instructions. Having placed their order, the customer must then hang around near the contradiction in anticipation of their order is ready for compilation. The additional difficulty in the food tune-up business is that restaurants are not realizing the efficiencies that would consequence from better request of technology in their day by day operations. Fast food business in a very spirited business and one way to stand out from competitors is through humanizing the production process where business process computerization can aid business development. The other problem with the modern organization is that the regulars are not clever to see the ingredients of the meals earlier than they place their order and moreover they only contain to disburse in favor of an ordering online.

We proposed an enjoin food through online system, in the first instance sketched for use in college buffet, but just as suitable in any food enjoin diligence. The main advantage of this system is that it very much removes the complexities form the enjoining progression for both the purchaser and the eatery. At what time the customer go to see and spend time with the enjoin webpage, they are dispense with an givenand-take and up-to-date menu, finished with all within easy reach options and dynamically alternating rate based on the fixed options. In the wake of later than building a selection, the object is then append their order, which the customer can analysis the attributes of at any time earlier than inspecting out. This gives immediate visual support of what was selected and protect that objects in the order are, actually what was calculated. This method also by a considerable amount temper the stack on the eating places end, as the absolute process of taking orders is controlled. Once an order is put in the particular location on the webpage, it is intruding into the database and then recovered, in lovely a great deal immediate by desktop functions on the restaurants end. Enclosed by this application, all object in enjoins are manifest, along with their equivalent options and conveyance details, in a succinct and easy to read the mode. This permits restaurant workers to rapidly set off from side to side the instructions as they are located and construct the needed objects with the smallest amount of hold up and indecision.

Enjoin food through online system is a generally transmitted information system that permit its users construct invocation for a particular repast and have the repast brought to their doorstep. This kind of system is usual for food restaurants. Consumer by means of Smartphone when the customer move toward to the restaurant, the saved categorize can be established by moving the Smartphone. The list of preferred preordered items shall be made known on the panel, and when long-established, order trip shall be printed for additional order dispensation. The resolution provides easy and well-located way to select pre-order contract form clients. The crucial reason why this system is built is because the restaurant is looking to expand their business to reach customers at a spacious scope, the customers do not have to eat in at the restaurant, and as a substitute the food will be brought to them. Online can be said to be anything that is associated or has links to the internet/world wide web, enjoining is movement that associate to asking another person to execute a task that will please you, conveyance is the action that involves another person or party bringing a object to your person or another person has enjoined by you or another party. This investigation will be centered on finding reality about serviceable website for enjoin food through online by looking into the framework, problem statement, aim, objective and literature review of the system

\section{RELATED WORK}

To rise above the restrictions of exceeding scheme, we recommend custom built for serviceable website for enjoin 
food through online. This revision lays away a skeleton for a new scheme to be urbanized and brought to the support for utmost consume and to generate an opportunity through the network wherever users can register on to our attendant and formulate a variety of whatever produce or food they similar to and consequently disburse via the internet. All over the system investigate, an in detail, revise of customer in sequence is conducted, for producing efficient necessity of the projected system. Information regarding the presented system is composed through numerous actuality-ruling techniques such as website trip and document evaluation, at the opening of this period. The data composed services information necessary the whole time comprehensive examination. A study on the modern system is performed based on the composed data. So, the user necessities of the proposed system are strong-minded. Towards the end of this stage, requirement design is formed as deliverable.

\section{PROBLEM STATEMENT}

As economic activity concerned with the processing of raw materials and manufacture of goods in factories are fast enlarging, people are try to obtain for more ways to acquire products with much simplicity and still preserve the cost efficiency. The trader's necessitate purchasing the products with the intention of put up for sale to end users. The laborintensive method of going to their local food sales outlets to acquire foodstuff is becoming outmoded and more tasking. Food can be prearranged from beginning to end the internet and imbursement completed without going to the eatery or the provisions retailer. So there is need for an extensive assortment of media hype and enabling shortest in order, dispensation and delivering of foodstuff in the course of online scheme. For this classification, there will be a system administrator who will have the constitutional rights to enter the list of options with current established prices.

\section{OUR CONTRIBUTION}

The proposed system is urban to handle ordering actions in hasty food eatery. It helps to evidence consumer submitted information. The scheme is supposed to wrap the following functions in order to maintain the restaurant's production method for achieving the objectives. This system is also contains the following features.

1. Precision in behavior of data. 2. The number of paper effort will be significantly condensed. 3. High-speed rate of business as in building the prepared food accessible and delivered on time. 4. Flexibility (i.e. it can be accessed at any time). 5. Improved storage and earlier recovery system. 6 . Errors in the intelligence will be significantly minimized. This system to permit the consumer to compose order, analysis order and compose changes previous to submitting their order and permit them compose payment through forestallment card as well, the system ought to be supported by the food derivation flavor and armed forces to keep the customers' faithfulness and happiness. However, broadly implementing the food ordering system possibly will source the incursion of employment due to the exclusion of waiters in restaurant trade. Even the system is significant to be implemented, yet there is still some danger in other factors such as a straight communication and restaurant plan perception, which need to be well thought-out for ensuring the sensation of the system. Make available boundary that allows endorsement and list of options to check boundary that shows customers' instructions aspect to face-end and kitchen staffs for delivering customers' orders for tackle that produce information to facilitate can be used for decision making. System should be easy to find the way, not clustered and simple to build an organizer, planned with professionals looking with search engine optimize ability and accessible 24hours. The system should also have a protected compensation prospect to defend their customers' credit cards in sequence, fast and keep track on instructions and sales record easily as well as produce a complete sales description, An instrument that allows the organization to transform the food in order such at the same time as consequences, include a new list of options and several others as able-bodied as apparatus for organization user, system set of choices and endorsement report. This will necessitate the input of essential data and record of speedy foodstuff ordering and liberation and then a statement is generated. This system helps to shrink stand in line issues for the period of hit the highest point hours, rapidity up food research and increase customer volumes. As a result, market divide up of fast food eatery can be boosted up and increases return of speculation for the shareholder.

Our contributions can be summarized as follow as.

1. On the way to permit the consumer to compose order, analysis order and compose changes previous to submitting their order and permit them compose payment through forestallment card or credit card or debit card.

2. To make available boundary that allows endorsement and list of options

3. To check boundary that shows customers' instructions aspect to face-end and kitchen staffs for delivering customers' orders

4. Tackle that produce information to facilitate can be used for decision making.

5. An instrument that allows the organization to transform the food in order such at the same time as consequences, include a new list of options and several others as able-bodied as apparatus for organization user, system set of choices and endorsement report.

\section{SYSTEM SPECIFICATION \\ 5.1 Design Standard}

The system is intended with a number of message indications on each web page that makes up the web application .These indications are distinct such as to construct a number of functionality that the request bare to gather progression and output data. Right of entry to these functionalities is made probable by the well intended user boundary which embodies several technologies such at the same time as AJAX (Asynchronous JavaScript and XML) to process data. The request is built in a modular form where these functionalities are built into modules.

\subsection{Output Specification}

This organization is intended in such a method that it proficiently provides output to the user quickly and in a healthy ordered behavior. The construct for the numerous output are construct accessible on the output web pages. Output can be relayed using the subsequent page modules. Products are exhibit output details for the inventory of food intricacy which are currently obtainable. Customers can expose to view productivity facts for the order description .which is based on the get results to the given query. Customer also displays the information about food details and about our organization. 


\subsection{Input Specification}

This system is intended to believe several input details proficiently from beginning to end contribution forms and customer clicks. The data captured from first to last the customer key strokes and clicks are established by exact modules on the scheme and relayed to the rear-end of the system for dispensation. Contribution is composed using the subsequent page modules. UI is used to imprison beginning user direction-finding in sequence and favorite in order which gives the system a technique of personalizing the page for the user on the subsequently excursion. Admin can imprison in sequence about the organizational people who reins satisfied and exhibit on the system

\subsection{Database specification}

The database system used to execute the rear-end of the system. Right of entry to the system was complete probable by a graphical boundary. In the database creation, need to create new database and the makeup of the data tables in the database which is very important for storing the all objects.

\subsection{Customer feedback}

After finishing ordering process the customer can present the feedback about the foods and also admin can view the feedback of the customers. By the use of feedback process the organization can measure the quality and quantity of their foodstuff.

\subsection{System model}

The makeup of the organization can be divided into three major rational mechanisms. The first constituent must present some form of tariff management, allowing the eatery to control what can be prearranged by clients. The second constituent is the mesh ordering scheme and provides the functionality for consumers to rest their order and provide all essential particulars. The third and final rational constituent is the tidy recovery scheme. Used by the restaurant to maintain way of all orders which have been located, this constituent takes be concerned of getting and showing order information, as well as informing instructions which have previously been practiced

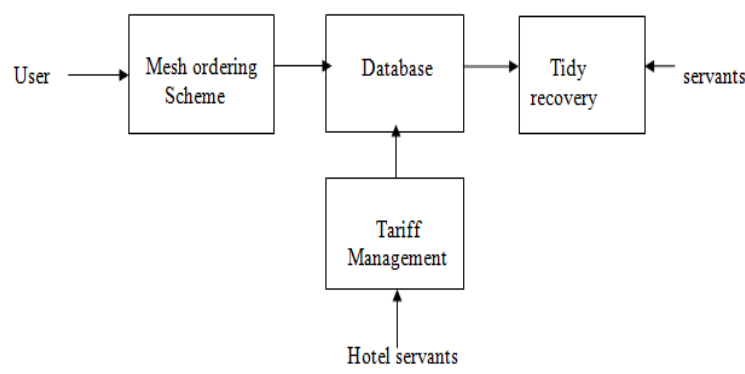

Fig 1. System model

\section{FUNCTIONAL REQUIREMENTS}

At the same time as know how to be seen in the structure model diagrammed over, each of the three system mechanism basically provides a cover of separation between the end user and the record. The inspiration after this separation is double. Firstly, allowing the end user to interrelate with the system during a rich boundary make available a much more pleasant user experience, mostly for the non-industrial users which will account for the best fraction of the classification's users. Besides, this separation layer also protects the honesty of the record by preventing users from attractive any action exterior those which the system is designed to hold. Because of this design model, it is necessary to specify accurately which purposes a user will be obtainable and these utilities are outlined below, clustered by constituent.

\subsection{The mesh ordering scheme}

Users of the mesh ordering scheme, namely eatery consumers, must be supplied the following functionality:As the objective of the scheme is to build the process of insertion and tidy as simple as possible for the consumer, the functionality offered from side to side the web enjoining system is controlled to that which is the greater part applicable to achieve the preferred mission.

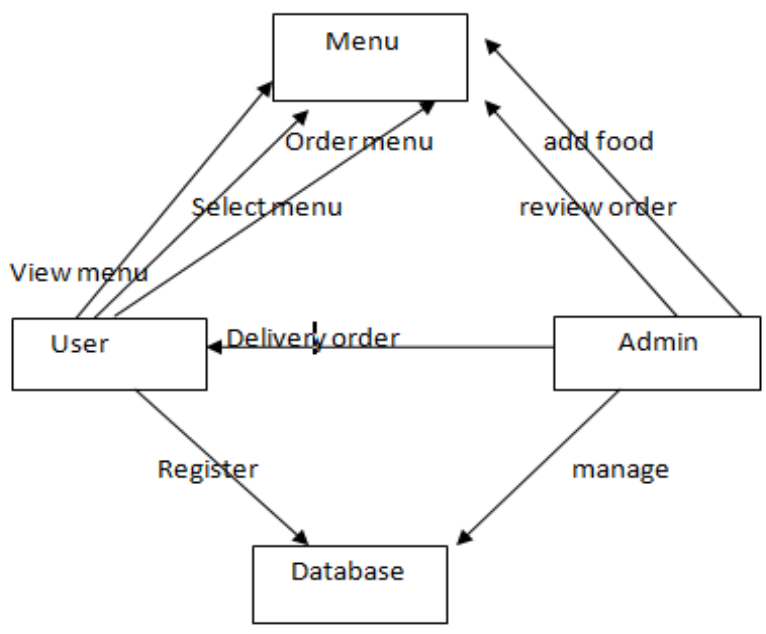

Fig 2. Mesh ordering system

All of the functions outlined exceeding, with the exceptions of account formation and management, will be used each time a consumer spaces a request. By not together with irrelevant functions, I am touching towards my objective of simplifying the enjoining procedure.

\subsection{Tariff management system}

The tariff management system will be available only to hotel servants and will, since the name proposes, permit them in the direction of supervising the list of options that is showed to users of the web enjoining scheme. The reason gives by the tariff management system presents consumer with the capability to, using a graphical boundary:

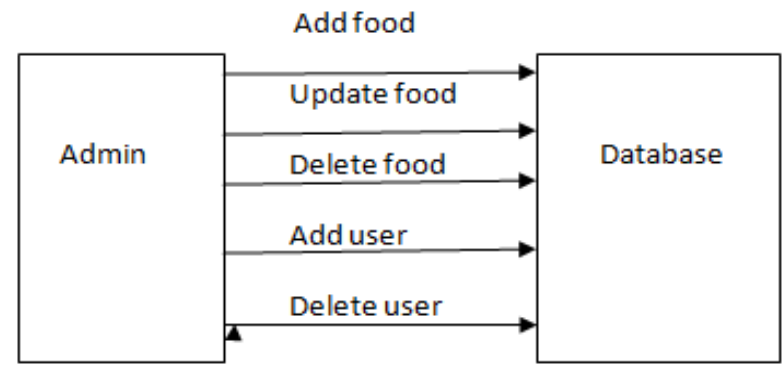

Fig 3. Tariff system 
It is projected that the functionality offered by this constituent will be individual of the primary belongings renowned by the eatery consumer, as they will have to exit from side to side it to construct their set of choices, etc. earlier than opening to essentially obtain instructions. Once the whole thing is originally configured, nevertheless, this constituent will possible be the smallest amount used, as set of choices updates usually do not happened with great incidence.

\section{Tidy recovery system}

Of the three components, the tidy recovery system is functionally the simplest. Like the tariff management scheme, it is intended to be used barely by hotel servants, and gives the following purposes: recover new instructions from the record. Display the instructions in an effortlessly understandable, graphical way. Blotch an orderly as having been processed and remove it from the list of dynamic instructions.

\section{USER INTERFACE SPECIFICATIONS}

Each of the scheme machinery will have their own exclusive boundary. These are described below.

\subsection{The mesh ordering scheme}

Users of the mesh ordering system will interconnect with the request from beginning to end sequence of simple forms. Each group of food has its own structure associated with it which presents a drop down set of choices for selecting which precise article from the group should be supplementary to the order and a sequence of test boxes and broadcasting buttons for selecting which options are to be integrated. Adding an article to the order is talented by a particular button click. Users pick which group of foodstuff they would similar to order, and therefore which form should be showed, by navigating a menu bar, an advance which be supposed to exist familiar in the direction of most users.

Entering liberation and imbursement deals is done in a related approach. The user is obtainable through a form and must complete the essential fields, which contain both falls down and content boxes, before inspection out and getting an authentication number. Single obsession significance noting here is that at whatever time possible falls down boxes and buttons were used over freeform input with the intention of both shorten the enjoining process and decrease the possibility of and SQL injection attempt.

\subsection{Tariff management system}

User interaction with the tariff management system is related to the mesh ordering systems. Users navigate a tree structure to discover the category, vendor, or exact food item that they would similar to change and after manufacturing their selection they are offered by means of a form which shows all of the present values and fields connected by with the intention of item, all of which can be changed or removed. The form also presents buttons which allow the addition of new values and field's .disparate the mesh enjoining scheme, however, most of the input here will be freeform, particularly in the form of content boxes, since there is no limited place of fields which might be added. This does not elevates a concern though, as input sanitation will be achieved, and the consumer, who is assumed to be an eatery worker, is less likely to be spiteful than a mesh consumer.

\subsection{Tidy Recovery System}

User interaction with the tidy recovery will be very easy. The application will automatically obtain latest orders from the database at usual intervals and display the delivery time, along with order numbers, in a board on the absent dispense side of the submission. To view the details of an order, the user must click on that order number, which will populate the right-hand panel with the details, displayed in an easy to read and navigate tree structure. This structure can instinctively be collapsed and extended to exhibit only the preferred information. Finally, once and order is practiced, the consumer ticks a single button, labeled "Processed", to ignore it from the directory of dynamic orders.

\section{NON-FUNCTIONAL REQUIREMENT}

Since the blueprint examples of the Online Ordering System are attractive much the standard for a web application, the non-functional requirements of the system are uncomplicated. Even though printed using Google Web Toolkit, the application is cross-compiled to JavaScript and HTML, together with a PHP backend, all of which are maintained by any reasonably well retained mesh server, though I would advised Apache2, and mainly the free XAMPP circulation. All of the relevance data is amassed in a PostgreSQL database, and consequently a PostgreSQL server must also be established on the horde processor. As with Apache2, this software is freely available and can be established and run under most in service schemes. The server hardware can be any processor competent of running both the record and web servers and managing the predictable traffic. Intended for an eatery that is not expectant to see much mesh traffic, or possibly doing only a incomplete test run, an average personal computer may be appropriate. Once the site starts generating more hits, though, it will likely be necessary to promote to a dedicated host to make sure proper performance. The accurate cutoffs force need to be strong-minded through a more detailed pressure testing of the scheme.

\section{SYSTEM EVOLUTION}

Since declared in the structure model, at the spirit of the whole enjoining scheme is the record. In fact, the scheme might be totally prepared using nothing but the database and an suitable shell utility, presuming that all consumers are fine-versed in SQL and like using it to order food. Though this would be a bit intense, it does demonstrate the position that the single part of the scheme which will stay relatively constant is the record. On the further hand, it is extremely credible that the other components will continue to progress with time. For instance, with the thriving reputation of mobile apps, I would like to create the mesh boundary available as a phone app as well. As well it may construct intelligence to at some point transfer the tariff management and tidy recovery systems to mesh, or constant cellular phone, apps as well, as some consumers may favor to use them as such. I am besides confident that if this scheme goes into genuine use, lot of requirements will take place for further features which I had not beforehand measured, but would be helpful to have. For this reason, I feel as though the request can be continually evolving, which I think about a very good thing. 


\section{CONCLUSION}

The development of serviceable website for enjoin food through online concerned many phases. The approach used is a top-down one focused on what first, then how and moving to consecutive levels of details. The first stage started with a detailed study of the troubles and prediction of ordering in foods. In the course of this study, many problems were discovered to have caught up the efficiency of the obtainable physical scheme. These problems, information requirements and behavior were documented and afterward used as the basis for system design, which instantaneously followed the first stage. The design phase was troubled primarily with the specification of the system essentials in behavior that best met the organization's business requirements. During this phase, strict observance was made on established software engineering principles and practices. It is hoped that effective completion of this software product would remove many problems exposed during systems examination.

\section{REFERENCES}

[1] PriyankaThakare, ReshamShinde, SushimitaSarkar,“ Design and Implementation of Digital Dining In Restaurants Using Android"International Journal of Advance Research in Computer Science and Management Studies Volume 2, Issue 1, January 2014.

[2] M. Firdouse Ali Khan, Swapna, "Design and Implementation of Ordering System for Restaurants", in International Journal of Engineering Research \& Technology (IJERT), Vol. 1, Issue 10, December- 2012.

[3] Wei Wing Chiew, Wen Jiun Yap, Soon Nyean Cheong, "Design and Development of Multi-Touchable ERestaurant Management System”, in 2010 International Conference on Science and Social Research (CSSR 2010), December 5 - 7, 2010, Kuala Lumpur, Malaysia.

[4] M.H.A. N. Ahmad, A.A. Mutalib H.A. Kadir, Wahab and M.F.M. Mohsin, "Implementation of network-based smart order system," International Symposium on Information Technology 2008 (ITSim 2008), pp. 1-7, 2008.
[5] Hashim,NikMohdZarifie and Ali,Nur Alisa and Ja'afar,AbdShukur and Mohamad, NajmiahRadiah and Salahuddin, LizawatiandIshak, Noor Asryran (2013) Smart Ordering System via Bluetooth. International Journal of Computer Trends and Technology (IJCTT), 4 (7). pp. 2253-2256.

[6] M. ErdiAyob, Ayob J., Mohd. Helmy A. Khairunnisa K., Wahab, M. IzwanAyob, M. AfifAyob "The Application of Wireless Food Ordering System," MASAUM Journal of Computing, Volume 1 Issue 2, September 2009,pp $178-183$.

[7] VarshaChavan,PriyaJadhav,SnehalKorade,PriyankaTel iProf.Mr.R.B.Anpat'Implementing Digital Restaurant and Inter-Restaurant Navigation Using Smart Phone",inInternational Journal of Computer Science and Mobile Computing,Volume 4,Issue 2 February 2015.

[8] Apurva Joshi, PrachiOke, NiranjanJadhav, AshutoshBhargave Prof. Mr. S. R Lahane, "Digital Ordering System for Restaurant using Android", in International Journal of Scientific and Research Publications, Volume 3, Issue 4, April 2013.

[9] Ayob J., Mohd. HelmyA.Wahab, Khairunnisa K., M. IzwanAyob, M. AfifAyob, M. ErdiAyob, "The Application of Wireless Food Ordering System", in MASAUM Journal of Computing, Volume 1 Issue 2, September 2009.

[10] Sun Guiling; Qingqing Song, "Design of the Restaurant Self-Service Ordering System Based on ZigBee Technology, "Wireless Communications Networking and Mobile Computing (WiCOM), 2010 6th International Conference on, vol., no., pp.1,4, 23-25 Sept. 2010.

[11] Jhabuawala Mustafa, SlatewalaAbdulquadir, NaikRiddhi, Kothari Radhika, "Touch \& Dine - A Multi- Touchable Restaurant System", in UACEE International Journal of Computer Science and its Applications - Volume 2: Issue 1. 